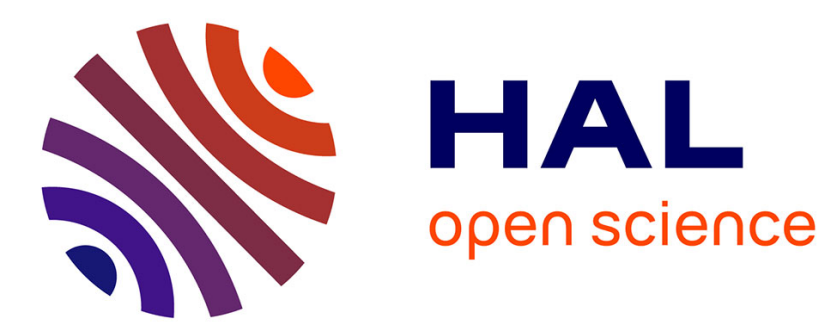

\title{
Anisotropy of the Anelastic Relaxation Associated with Interstitial Carbon in Nickel
}

H. Numakura, K. Kashiwazaki, M. Koiwa

\section{To cite this version:}

H. Numakura, K. Kashiwazaki, M. Koiwa. Anisotropy of the Anelastic Relaxation Associated with Interstitial Carbon in Nickel. Journal de Physique IV Proceedings, 1996, 06 (C8), pp.C8-127-C8-130. 10.1051/jp4:1996825 . jpa-00254633

\section{HAL Id: jpa-00254633 https://hal.science/jpa-00254633}

Submitted on 1 Jan 1996

HAL is a multi-disciplinary open access archive for the deposit and dissemination of scientific research documents, whether they are published or not. The documents may come from teaching and research institutions in France or abroad, or from public or private research centers.
L'archive ouverte pluridisciplinaire HAL, est destinée au dépôt et à la diffusion de documents scientifiques de niveau recherche, publiés ou non, émanant des établissements d'enseignement et de recherche français ou étrangers, des laboratoires publics ou privés. 


\title{
Anisotropy of the Anelastic Relaxation Associated with Interstitial Carbon in Nickel
}

\author{
H. Numakura, K. Kashiwazaki and M. Koiwa \\ Department of Materials Science and Engineering, Kyoto University, Yoshida Hon-Machi, Sakyo-ku, \\ Kyoto 606-01, Japan
}

\begin{abstract}
The behaviour of interstitial $\mathrm{C}$ atoms in $\mathrm{Ni}$ has been studied by low-frequency internal friction measurements using single crystal specimens. The magnitude of the relaxation peak, which appears at $520 \mathrm{~K}$ for $f=1 \mathrm{~Hz}$, is the largest when the axis of shear stress is $\langle 111\rangle$, while the peak is virtually absent when the stress axis is $\langle 100\rangle$. This behaviour can be accounted for by considering pairs of $\mathrm{C}$ atoms occupying the 2 nd neighbour octahedral sites. The kinetics of the relaxation is discussed by assuming the relaxation is due to the reorientation of the 2 nd neighbour pairs through the 3rd neighbour configuration.
\end{abstract}

\section{INTRODUCTION}

Anelastic methods have made significant contributions in the studies of the behaviour of interstitial solute atoms in metals, particularly for bcc metals. In fcc metals, interstitial atoms occupying the tetrahedral or octahedral sites cannot give rise to anelastic relaxation because the symmetry is the same as the host lattice. However, when solute atoms form complexes of lower symmetry, they may produce anisotropic distortion of the lattice and undergo stress-induced reorientation. In fact, a number of anelastic effects have been reported for fcc metals containing interstitial solutes [1], most of which have been attributed to interstitial-interstitial ( $\mathrm{i}-\mathrm{i}$ ) and interstitial-substitutional ( $\mathrm{i}-\mathrm{s}$ ) atom pairs.

A typical example is the internal friction peak observed for dilute $\mathrm{Ni}-\mathrm{C}$ alloys [1]. The height of the peak varies as the square of the $\mathrm{C}$ concentration [2], indicating that the peak is associated with pairs of $\mathrm{C}$ atoms. A magnetic relaxation effect corresponding to the internal friction peak has also been observed [3]. Diamond and Wert [4] studied this effect by anelastic and magnetic measurements and reported that the activation parameters are in good agreement with the diffusion behaviour of $\mathrm{C}$ at high temperatures. They discussed the results on the assumption that the relaxation is caused by the reorientation of $\mathrm{C}$ atom pairs occupying the 1st neighbour octahedral sites. The aim of this investigation is to determine the configuration of $\mathrm{C}$ pairs from the anisotropy of the relaxation magnitude, and to clarify the atomistic mechanism of the relaxation process.

\section{EXPERIMENTAL PROCEDURE}

Single crystals of pure nickel were prepared from the raw material of $99.97 \%$ purity using an inductionmelting Bridgman furnace. Square bar specimens, typically $0.7 \mathrm{~mm} \times 0.7 \mathrm{~mm}$ in cross-section and 50 $\mathrm{mm}$ in length, were cut out of the crystals. The orientation of the bars was chosen to be nearly parallel to [100], [411], [211] or [111]. The specimens were loaded with $C$ (about 1 at.\%) by annealing in a flow of $\mathrm{CH}_{4}$ and $\mathrm{H}_{2}$ of atmospheric pressure at $1050^{\circ} \mathrm{C}$ for $4.8 \mathrm{~h}$. After this treatment, the specimens were quenched to $0^{\circ} \mathrm{C}$. The concentration of $\mathrm{C}$ in solution was estimated from the increase in electrical resistivity of the specimen, which was measured at $77 \mathrm{~K}$, using the value of the specific resistivity, $\Delta \rho=33$ $\mathrm{n} \Omega \mathrm{m} /$ at.\% C [5].

Internal friction was measured using an inverted torsion pendulum by the free-decay method at frequencies of about $1 \mathrm{~Hz}$ with the surface shear-strain amplitude of $4 \times 10^{-6}$. Measurements were made in an atmosphere of $\mathrm{He}$ of about $100 \mathrm{~Pa}$ over the temperature range from $300 \mathrm{~K}$ to $640 \mathrm{~K}$ with a heating rate of $1 \mathrm{~K} / \mathrm{min}$. 


\section{RESULTS}

Figure 1 shows the internal friction $Q^{-1}$ and the square of the frequency $f$ of the [211]-oriented specimen measured before and after C-loading. The $Q^{-1}$ versus temperature $T$ curve before loading shows only a broad spectrum, while the curve after $\mathrm{C}$ loading (1.15 at.\%) exhibits a maximum at $520 \mathrm{~K}$. This peak is identified as the same internal friction peak observed in polycrystalline specimens by previous researchers and also in the present work [6]. The $f^{2}$ versus $T$ curves show a marked drop centred at 400$450 \mathrm{~K}$, which must be due to the motion of magnetic domain boundaries under stress, i.e. an essentially the same effect as the $\Delta E$ effect observed for the Young's modulus [7]. The $f^{2}$ curve after C-loading changes its slope abruptly from positive to negative at $610 \mathrm{~K}$. This is related to the ferromagnetic-paramagnetic transition; the transition temperature, which is $634 \mathrm{~K}$ in pure $\mathrm{Ni}$, is lowered by the addition of $\mathrm{C}$ at a rate of $\sim 30 \mathrm{~K}$ per at.\% C [8]. The $Q^{-1}$ and $f^{2}$ curves of the other specimens before C-loading were essentially similar to those of the [211] specimen.

Figure 2 summarises the results after C-loading for the four orientations. The carbon concentrations are approximately equal to each other, viz. $0.99,0.98,1.15$ and 1.22 at.\% for the [100], [411], [211] and [111] specimen, respectively. The height of the peak at $520 \mathrm{~K}$ depends strongly on the crystal orientation; the peak is almost absent for the [100] specimen, while it attains $(2 \sim 3) \times 10^{-3}$, subtracting the background, for the [111] specimen. The magnitude of the relaxation of the shear compliance, $\delta G^{-1}$ $=\Delta G^{-1}$, has been determined for each specimen by taking the twice the peak height as the relaxation strength $\Delta$ and calculating the orientation-dependent shear compliance, $G^{-1}$, from the anisotropic elastic constants of $\mathrm{Ni}$ at $520 \mathrm{~K}$ [9]. The values of $\delta G^{-1}$ thus obtained are plotted in Fig. 3 against the orientation factor $\Gamma$, which is defined as

$$
\Gamma=\gamma_{2}^{2} \gamma_{3}^{2}+\gamma_{3}^{2} \gamma_{1}^{2}+\gamma_{1}^{2} \gamma_{2}^{2},
$$

where $\gamma_{i}$ are direction cosines between the stress axis ( $=$ the bar axis) and the crystal axes. The relaxation magnitudes are normalised to 1 at. $\% \mathrm{C}$, assuming that the peak height is proportional to the square of the $\mathrm{C}$ concentration. Taking into account the range of possible errors, which come mostly from uncertainties in background subtraction, it is concluded that $\delta G^{-1} \approx 0$ at $\Gamma=0$ and increases proportionally with $\Gamma$.

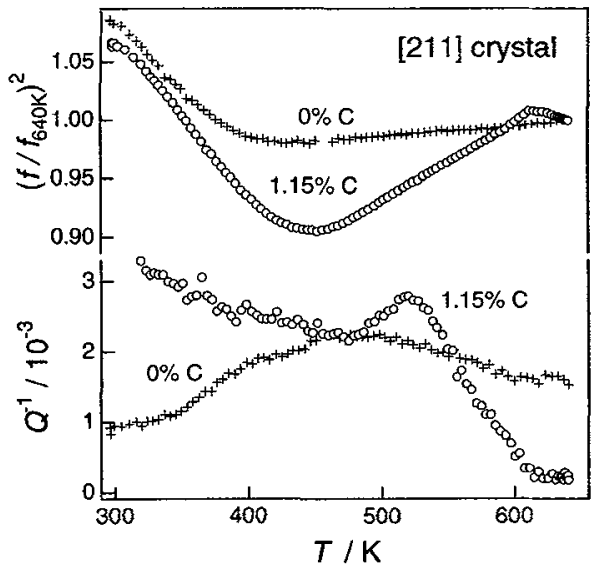

Figure 1: Internal friction and the square of the frequency of Figure 2: Internal friction of the specimens of different orithe [211] specimen before and after loading 1.15 at. \% $\mathrm{C}$.

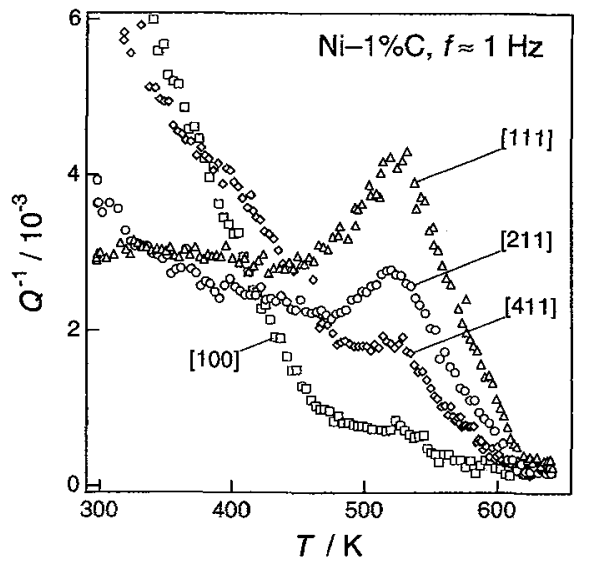

entations loaded with $\sim 1$ at. \% C.

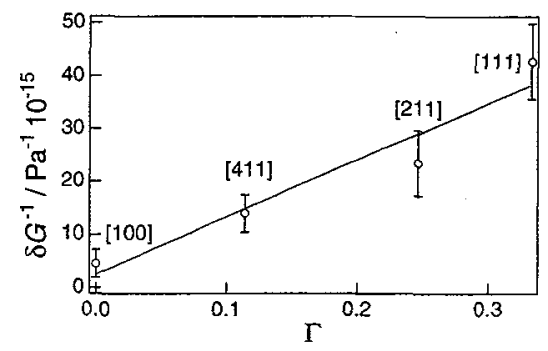

Figure 3: Orientation dependence of the relaxation magnitude of the peak at $520 \mathrm{~K}$. 


\section{DISCUSSION}

The magnitude of the peak at $520 \mathrm{~K}$ measured in torsion has been found to increase with increasing $\Gamma$. For cubic crystals, the anisotropy of the relaxation under shear stress is generally expressed as

$$
\delta G^{-1}=\delta S^{\prime} 2 \Gamma+\delta S(1-2 \Gamma)
$$

where $\delta S^{\prime}$ and $\delta S$ are relaxations of the shear compliances $S^{\prime} \equiv 2\left(S_{11}-S_{12}\right)$ and $S \equiv S_{44}$, respectively. The result of the present experiment implies that the relaxation consists only of $\delta S^{\prime}$. According to the selection rule for anelastic relaxation [10], defects that give rise to $\delta S^{\prime}$ but not to $\delta S$ are those of tetragonal or $\langle 100\rangle$ orthorhombic symmetry. Table 1 shows some close-neighbour configurations of a pair of interstitial atoms occupying octahedral sites. Among the pairs listed in the table, only the 2nd neighbour configuration conforms to the observed anisotropy. Therefore, the peak is attributed to reorientation of the 2 nd neighbour $\mathrm{C}-\mathrm{C}$ pairs. A similar result was reported for the internal friction peak associated with $\mathrm{C}$ in fcc Co: Mah and Wert [11] found that the relaxation is almost entirely of $\delta S^{\prime}$, and discussed the mechanism in preference for the 2 nd neighbour $\mathrm{C}-\mathrm{C}$ pairs as the defect responsible for the relaxation.

Interstitial $\mathrm{C}$ atoms increase the lattice parameter $\mathrm{Ni}$ [12], and are thus centres of dilatation. the elastic interaction energy between such dilatation centres in cubic crystals is given by anisotropic elasticity theory [13] as

$$
\begin{gathered}
E=\frac{15}{4 \pi} \frac{\left(C_{11}-C_{12}\right) / 2-C_{44}}{[3(1+v) /(1-v)]^{2}} \frac{\Delta V_{1} \Delta V_{2}}{r^{3}} \Lambda, \\
\Lambda=\alpha_{1}^{4}+\alpha_{2}^{4}+\alpha_{3}^{4}-\frac{3}{5},
\end{gathered}
$$

where $C_{i j}$ are elastic stiffness constants, $v$ is Poisson's ratio, $\Delta V_{1}$ and $\Delta V_{2}$ are the volume expansions associated with the two defects, $r$ is their separation, and $\alpha_{i}$ are direction cosines between the pair axis and the crystal axes. In the present problem, i.e. two identical defects in $\mathrm{Ni}$, the second factor in Eq. 3 is negative since the shear anisotropy in $\mathrm{Ni}$ is such that $\left(C_{11}-C_{12}\right) / 2<C_{44}$, and the third factor is positive since $\Delta V_{1}=\Delta V_{2}$. The last factor $\Lambda$ varies from $2 / 5$ for the pair axis parallel to $<100>$ to $-4 / 15$ for $<111>$. Therefore, $E$ is negative for a pair aligned in $\langle 100\rangle$, thus interacting attractively, while it is positive for a pair in $\langle 111\rangle$, interacting repulsively. The magnitude of $E$ has been calculated for the pairs in Table 1 using the elastic constants of $\mathrm{Ni}$ at $520 \mathrm{~K}$ [9] and the volume expansion due to $\mathrm{C}$ in $\mathrm{Ni}, \Delta V=8.4 \AA^{3}$ [12]; the last column of the table shows the results. The interaction energy is of substantial magnitude only for the 1 st and the 2 nd neighbour pairs, the former being positive and the latter negative. The 2 nd neighbour pair is thus energetically favourable over the other configurations.

Discussion on the kinetics of the relaxation requires a detailed analysis, because a 2 nd neighbour octahedral interstitial pair cannot reorient by a single atomic jump. Figure 4 shows the geometry of the interstitial sites around an interstitial atom on an octahedral site. Since the 1st neighbour configuration is unfavoured, it is reasonable to assume that the reorientation of the $2 \mathrm{nd}$ neighbour pair is achieved by the jumps of $C$ atoms leading to the changes in the configuration as $2 \mathrm{nd} \rightarrow 3 \mathrm{rd} \rightarrow 3 \mathrm{rd} \rightarrow 2 \mathrm{nd}$, as indicated by the arrows in the figure. If this is the case, relaxations associated with the 3rd neighbour pair (a $<110>$ monoclinic defect) may occur, viz. one $\delta S^{\prime}$ and two $\delta S$ relaxations, in addition to the one $\delta S^{\prime}$ relaxation due to the 2nd neighbour pair. However, the observed anisotropy indicates that $\delta S$ relaxations are very small in magnitude. In what follows, we discuss solely the kinetics of the two $\delta S^{\prime}$ relaxations.

Following the general theory of kinetics of anelastic relaxation $[14,15]$, we have derived the expressions for the rates of the $\delta S^{\prime}$ relaxations. The final result is

$$
\tau_{ \pm}^{-1}=4 w_{23}+3 w_{33}+w_{32} \pm\left[\left(4 w_{23}-3 w_{33}-w_{32}\right)^{2}+16 w_{23} w_{32}\right]^{1 / 2}
$$

Table 1: Configurations of interstitial atom pairs occupying octahedral sites in the fcc lattice, referred to one interstitial atom located at the origin. $a$ is the lattice parameter, $n$ is the number of equivalent orientations and $E$ is the elastic interaction energy between two C atoms in Ni at $520 \mathrm{~K}$.

\begin{tabular}{ccccrr}
\hline Shell number & Relative position & Distance / $a$ & Defect symmetry & $n$ & $E / \mathrm{eV}$ \\
\hline 1 & $<1 / 21 / 20>$ & 0.707 & $<110>$ orthorhombic & 6 & 0.092 \\
2 & $<100>$ & 1 & tetragonal & 3 & -0.130 \\
3 & $<11 / 21 / 2>$ & 1.224 & $<110>$ monoclinic & 12 & 0.018 \\
4 & $<110>$ & 1.414 & $<110>$ orthorhombic & 6 & 0.011 \\
5 & $<3 / 21 / 20>$ & 1.581 & $<100>$ monoclinic & 12 & -0.018 \\
6 & $<111>$ & 1.732 & trigonal & 4 & 0.017 \\
\hline
\end{tabular}




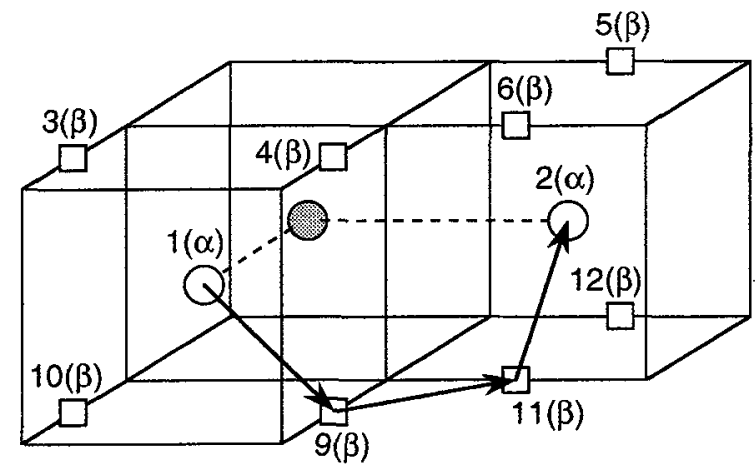

Figure 4: Reorientation of a 2 nd neighbour $i-$ $i$ pair in the fcc lattice through the two 3 rd neighbour positions. The numbers attached to each site in the figure denote the orientation labels [14] for a tetragonal defect (referred to as $\alpha$ ) and for $a<110>$ monoclinic defect $(\beta)$.

where $w_{m n}$ are the jump rates of an interstitial atom from the $m$-th neighbour site to the $n$-th neighbour site. The fact that both atoms in a pair can jump with equal probabilities has been taken into account. The theory predicts that the relaxation spectrum is composed of two Debye peaks, the relaxation times of which are given above. Further discussion of the relaxation spectra is difficult at the moment because it requires the knowledge of the relaxation magnitudes, which is governed by the lattice distortion around the defects involved.

Let us briefly examine a special case where $w_{23} \approx w_{33} \approx w_{32} \equiv w$. Equation 5 is then reduced to $\tau_{+}{ }^{-1} \approx 12 w$ and $\tau_{-}^{-1} \approx 4 w$. The pre-exponential factor and the activation energy of the jump frequency $w$ are obtainable from diffusion data at high temperatures [16], using the relation $D=w a^{2}$, as $w_{0}=2.42 \times$ $10^{14} \mathrm{~s}^{-1}$ and $Q=1.54 \mathrm{eV}$. From these parameters, the peak temperatures of the two relaxations for $f=$ $1 \mathrm{~Hz}$ are predicted to be $529 \mathrm{~K}$ and $547 \mathrm{~K}$, which are in reasonable accord with the experimental observation. Moreover, the magnitudes of the relaxation rate, viz. $12 w$ and $4 w$, agree well with the relaxation rate determined experimentally by Diamond and Wert [4],

$$
\tau^{-1}=2.8 \times 10^{15} \exp (-1.51 \mathrm{eV} / k T) \mathrm{s}^{-1} \text {. }
$$

In a separate experiment on polycrystalline specimens by the present authors [6], the peak has been found to be broader than the Debye peak of this relaxation time by about $10 \%$. This feature may be explained in terms of the presence of two components with relaxation rates close to each other.

\section{Acknowledgements}

The authors are grateful to Dr T. Takasugi (IMR, Tohoku University) for preparing single crystals, and to $\mathrm{Mr} \mathrm{K}$. Murakawa (Kyoto University) for his help in specimen preparation.

\section{References}

[1] Nowick, A. S., and Berry, B. S., Anelastic Relaxation in Crystalline Solids (Academic, New York, 1972) Chapter 11.

[2] Tsien, C., Sci. Sinica 10 (1961) 930-937.

[3] Wuttig, M., and Birnbaum, H. K., Appl. Phys. Lett. 7 (1965) 320-323.

[4] Diamond, S., and Wert, C., Trans. AIME 239 (1967) 705-709.

[5] Swartz, J. C., J. appl. Phys. 42 (1971) 1334-1335.

[6] Kashiwazaki, K., Master thesis (Graduate School of Engineering, Kyoto University, Kyoto, 1996).

[7] Bozorth, R. M., Ferromagnetism (Van Nostrand-Rheinhold, Princeton, 1951).

[8] Schwerer, F. C., J. appl: Phys. 40 (1969) 2705-2707.

[9] Alers, G. A., Neighbours, J. R., and Sato, H., J. Phys. Chem. Solids 13 (1960) $40-55$.

[10] Nowick, A. S., and Heller, W. R., Adv. Phys. 14 (1965) 101-166.

[11] Mah, G., and Wert, C., Trans. AIME 242 (1968) 1211-1216.

[12] Zwell, L., Fasiska, E. J., Nakada, Y., and Keh, A. S., Trans. AIME 242 (1968) 765-766.

[13] Leibfried, G., and Breuer, N., Point Defects in Metals I (Springer, Berlin, 1978) Chapter 4.

[14] Nowick, A. S., Adv. Phys. 16 (1967) 1-47.

[15] Nowick, A. S., J. Phys. Chem. Solids 31 (1970) 1819-1826.

[16] Cermak, J., and Mehrer, H., Acta metall. mater. 42 (1994) 1345-1350. 